\title{
NDSI 2005 Highlights Latest Advancements in Nanoscience and Technology
}

The 2nd Conference on Nanoscale Devices and System Integration (NDSI 2005), sponsored by the National Science Foundation, the IEEE Nanotechnology Council, the Information Storage Industry Consortium, and the Nanotechnology Foundation of Texas, was held in Houston, Texas, April 4-6, 2005. Designed as a highly interactive meeting, NDSI 2005 served as an open forum to evaluate the latest achievements in nanoscience and technology. As emphasized by co-chair Sakhrat Khizroev (Florida International University), "One of the goals of the conference is to engage the attendees in a productive discussion, forge new alliances, and identify critical research areas."

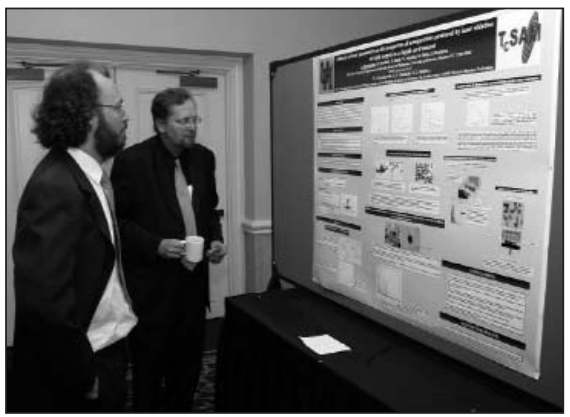

Adriano Cavalcanti (left) and Vitor Baranauskas (right) discuss nanomagnetics research being conducted at the Texas Center for Superconductivity and Advanced Materials.

The conference was organized in a singlesession format, with all invited speakers representing major academic research centers and industrial research and development (R\&D) facilities. Contributed work was showcased at poster sessions. The conference attracted more than 150 attendees from around the world and was locally hosted by the Cullen College of Engineering at the University of Houston.

The focus of the conference was novel fabrication technologies, devices, and nanoscale integrated systems that are continuously emerging as a result of various nanotechnology initiatives in chemistry, chemical engineering, physics, electrical engineering, materials science and engineering, biomedical engineering, and robotics.

Among the highlights of the conference were a number of talks by leading scientists and engineers, who presented the latest results and offered their perspectives on the future of nanotechnology. C. Marrian (IBM
Almaden Research Center) gave a critical overview of the role and potential of nanotechnology research in the semiconductor industry. S.V. Sreenivasan (Molecular Imprints) demonstrated step-and-flash imprint lithography, including the highfidelity replication of ultrasmall objects such as $5 \mathrm{~nm}$ carbon nanotubes. Imprint technologies are often touted as a potential replacement for current optical lithographies. H. Smith (Massachusetts Institute of

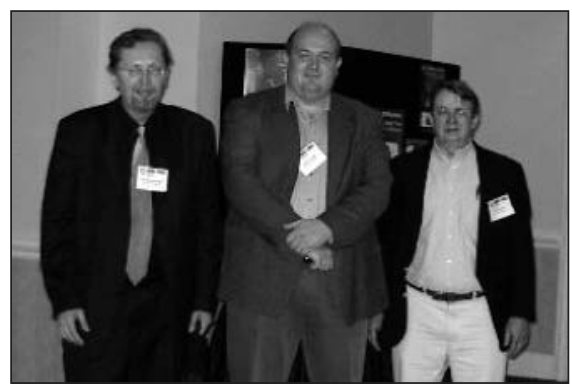

(Left to right): Vitor Baranauskas, Dmitri Litvinov (NDSI 2005 co-chair), and Jack Wolfe (NDSI 2005 local chair).

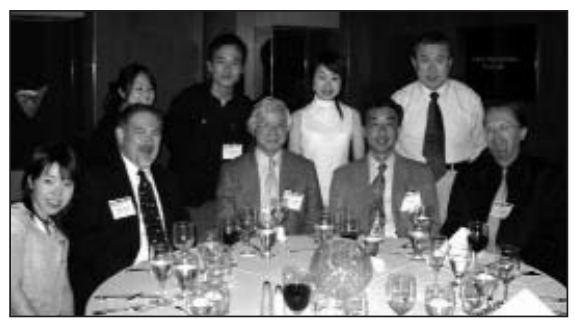

NDSI 2005 attendees enjoy dinner at the world-renowned Cullen Hall of Gems and Minerals in Houston, Texas.

Technology) presented advancements in zone-plane-array lithography, which aims to achieve nanometer accuracy and resolution. H. Linke (University of Oregon) presented research on power generation from thermal sources. R. Baughman (University of Texas at Dallas) gave an overview of various applications of carbon-nanotube yarns developed by his team. Impressive results in the field of onchip all-silicon nanophotonics were presented by M. Lipson (Cornell University). T. McDaniel (Seagate) discussed heatassisted nanomagnetic data storage systems. S. Hong (Samsung) described a resistive-probe data storage technology. V. Baranauskas (Universidad Estadual de Campinas, Brazil) demonstrated a high- performance implantable prosthesis based on nanocrystalline diamond ceramics. Groundbreaking results on the utilization of gold nanoparticles for cancer treatment and biosensing were presented by J. West and N. Halas (Rice University), respectively. R. Cowburn (Imperial College London) and W. Porod (University of Notre Dame) presented pioneering work on nanomagnetic logic devices that if fully developed and commercialized, could revolutionize integrated-circuit electronics. M. Sitti (Carnegie Mellon University) presented his latest work on implementation of gecko-foot-like fibrillar adhesives.

The conference featured an evening panel discussion on "Nanotechnology:

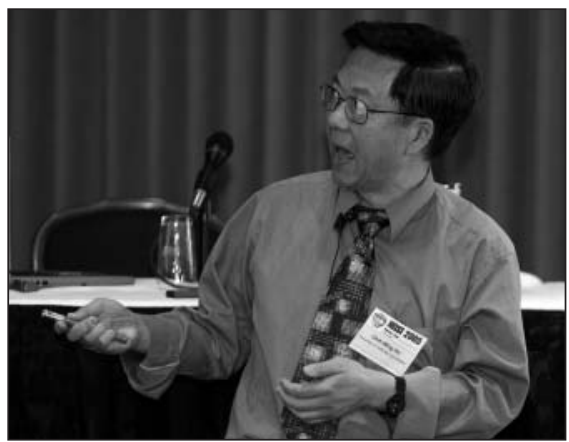

Chih-Ming Ho of the University of

California at Los Angeles presents his research on biologically inspired nanorobotics.

Present and Future Challenges," held on April 5, where representatives from several funding agencies and industry outlined their views on present opportunities and prospects for various nanotechnology initiatives. In addition to the discussion, panelists D. Herr of the Semiconductor Research Corporation, L. Madsen of the National Science Foundation, C. Marrian of IBM Almaden, and M. Stone of the Defense Advanced Research Projects Agency answered many questions from the audience.

The financial contributors to the conference included Veeco Instruments, RHK Technologies, the Texas Center for Superconductivity and Advanced Materials, and the Nanosystems Manufacturing Center at the University of Houston. Selected papers presented at the conference will be published in a special NDSI 2005 issue of IEEE Transactions on Nanotechnology.

DMITRI LITVINOV NDSI 2005 Co-chair 